\title{
Competitive activity indicators in handball. Statistics, analysis, forecast
}

\author{
Alexey Popovich*, Lyudmila Bezukladnova, Alexander Bezukladnov, Elena Goncharova \\ Ural Federal University Named After the First President of Russia B. N. Yeltsin, 620002, Yekaterinburg, Russia.
}

\begin{abstract}
The article deals with competition in modern sports. It reached its maximum. The competition is conducted within the limits of thousandths of a second, hundredths of a point, one point and a ball. Therefore, success comes to those teams that carefully prepare for matches. They should prepare physically, functionally and analytically, analyzing the opponent's game. The research of specialists ' work and review of materials published in domestic and foreign publications are carried out. The most significant analysis indicators of the competitive activity in handball are highlighted. The analysis of games in modern sports is insufficient and requires additional research on this problem.
\end{abstract}

\section{Introduction}

Handball is one of the most popular sports in the world. More than two hundred countries are members of the international Handball Federation. Handball develops through scientists and researchers as well as through the players. They make researches, analytics, game stats, analyzing the technical and tactical mistakes of the teams in matches.

In the USSR, handball was actively developed, as well as conducting scientific researches. They contributed to the growth of the number of Soviet handball players who became Olympic and World Champions several times. Among them are E. Ivakhin, N. Khomutov, L. Latyshkevich, A. Yevtushenko, V. Igantyeva, U. Makarov, A. Ratianidze, N. Klusov, V. Kontvainis, I. Turchin.

The development and analysis of competitive activity are at the center of specialists ' attention. The match and the tournament result depends on them. But generally accepted analytical standards do not fully reflect the course of the match. They fix the technical techniques of the players, but miss the tactical interactions of the opposing team and its other sides [2].

\section{Materials and methods}

This article reviews the colleagues' publications and discuss the problem of predicting the upcoming game.

When viewing the game, experts pay attention to the tactical structure of the opponent's game, team behavior, and player interaction. Descriptive analysis identifies the number and variety of player movements in different game schemes and variants. An important point is the" tactical " behavior of the player in accordance with the situations and game restrictions. If attacking actions in handball are carried out in conditions of discomfort (the angle of attack and active opposition of the defender, the distance to the goal), it is better to pay attention to their effectiveness and implementation. What is the effectiveness of the player at different time periods of the match, at a particular position of the player on the court, with active external influence from the defender [2].

We analyzed various information collection systems and indicators of athletes ' game activity during the match. The individual technical indicators analysis is carefully recorded. It is done for determining the title of the best in a particular category, correcting the training process for improving the result of the team's game and increasing the individual skill of the athlete [3].

In this review of the game activity analysis, we indicated in the table the data of specialists from Russia, Belarus, Asia, and Europe. The information is provided by the authors V. I. Ignatieva, V. I. Thorev, D. Milanovich, M. V. Porokhovskaya, M. V. Zhi-yar, S, V. Goncharuk, I. I. Boyko, V. S. Yakimovich, O. S. Abdalimov, A. Karastergiosu, Karol Gryko, Skufas Dimitrios, Murat Bilge, and Willian Ferrari.

In this article, we made a selection based on the published research of the above authors, analyzing handball, leading statistics of technical and tactical actions of players that affect a very wide range of the game.

Ignatieva V. Ya., Thorev V. I. [4], who for many years were part of the complex scientific group at the national teams of the USSR and the Russian Federation, emphasize that the control of competitive activity is aimed at assessing the level of the player skill. Forms of control are very different from code recording, voice tracking, video recording.

\footnotetext{
${ }^{*}$ Corresponding author: alex1957popovich@mail.ru
} 
Currently, video recordings with software are the most widely used. The authors consider the main indicators to be technical and tactical actions related to performance, efficiency and activity during the game.

When calculating the total number of passes, ball catching techniques, we can make a plan for the training load on the players' positions, create model characteristics of the game activity. It is better to record only those techniques and actions that led to a significant game situation improvement. A successful action counts as a "positive" for the player, and a failed action counts as a "passive". Disruption of the attack, the pace, dynamics of attacking actions, the speed of its development - this is a minus for the attacker. Defensive actions are evaluated in the same way. If the defense did not allow for a high-speed attack, its development was suspended - this is a plus for the defense. The creation of a numerical advantage by the opponent in one of the positions, regardless of its implementation, is a minus.

V. I. Thorev identified 12 attacking components with a certain significance coefficient and 7 components in defense. As a rule, individual evaluation actions are dominant. We can solve problems based on individual skill in attack, but we can't do it in defense. Only by interacting with partners you can successfully defense your own goal. A special role is assigned to the goalkeeper.

\section{Results and discussion}

If we have statistics on the individual players' game, we can get an information of the team's effectiveness or inefficiency as a whole. Therefore, it is possible to build conditional index models for each player, which are a "road map" to victory. One of the variants of the match forecast for the upcoming game was made by V. I. Thorev. But there are no ways of achieving a positive result.

Competitive activity indicators of 16 national teams, participating countries of the XIII European women's handball CHAMPIONSHIP held in France in December 2018, were statistically studied. 12 indicators of attack quantitative parameters were observed. They are: occupied space, the number of shots on goal, the number of abandoned goals, number of shots and goals scored from various positions, extreme, mid-range and a nine-meter line, the number of goals from counter-attacks, putting the ball quick.

Defensive actions include the taken position, the number of conceded goals, the number of ball grabs by defenders, blocking the ball, capture-warning, violation followed by removal for 2 minutes, the "save" of the goalkeeper, the number of shots that reached the goal and the goalkeeper, the percentage of goals scored by the goalkeeper, the stability of defensive actions. There are more than two dozen elements of collective tactics between productive and nonproductive teams. A team spends up to 25 seconds on a positive attack [5]. Special attention was paid to throws from the front line and "delivery" of the ball to this zone.

Handball in its evolutionary development provides analysts with a wide variety of information. The innovations of the international handball Federation in the rules of the game in 2001-2005 made the game faster and more dynamic, increase the pace and effectiveness of matches. This affected the technical and tactical training of handball players as well as the physical, functional state, and anthropometry.

The most effective actions are performed by players who are in optimal physical and functional condition. In a positional attack, a special role is assigned to the welterweights and the "center", and in a quick breakout and counterattack, the front line players are more important. Balance is achieved in the team's game.

It was interesting to get acquainted with the information of colleagues after the end of the XXIII World Cup and compare the implementation of prospects in the games of the XXIV world Cup. V. Thorev analyzed the most frequently used evaluation criteria in handball by analysts and compared them with the best national teams in the world. Along with BMI (body mass index), age, the number of games played for the national team, the number of goals scored per game, the number of shots, the number of goals and shots made from various positions and standards, counterattacks, and assists were also taken. Also actions in defense, and "saves" of the goalkeeper, which we reflected in the tab-face. We see the Russian national team among the winners of the XXIV world Cup in handball, but the list of competitive activity indicators does not reflect many important elements of the game that affect the result [6].

Here is how conducts competitive activity analysis Professor O. H. Abdalimov from Uzbekistan.

List of individual technical and tactical actions:

- effective shots from short, medium and long distances.

- after beat, counter, quick center.

- assist, penalty 7 meters, removal of the player.

In case of unsuccessful actions, it is described in more detail:

- goalie, bar, past, block indicates the distance to the goal, as well as the loss of the ball catching, passing, driving, running.

However, game actions without the ball are not reflected in the analysis.

In defense, the total number of attacks to the number of interrupted enemy attacks. The intensity of the match is vaguely estimated. It is not specified at the "expense" of what actions in the defense is an external impact on the opponent. The number of "single combats" in defense is also not reflected.

As a rule, when analyzing the competitive activity of the strongest teams in Europe, experts classify the five "indicators" that are used most often. These are 9-meter, 7-meter penalties, mistakes made during the game. Control and methods for determining the effectiveness of attacks in time intervals from 3-20 seconds. The number of technical and tactical actions and their effective implementation are the most informative. 
My colleague Boyko I. from Vitebsk University specializes in the analysis of attacking actions in handball. In our opinion, this approach is one-sided.

Interesting work is presented by Greek handball specialists, such as Skoufas Dimitrios [7]. After analyzing five women's handball world cups and systematizing the game actions that affect the result in the game, he came to the same conclusions as we did. The highest factor is won single combats and the increasing role of physical training, strength, speed, coordination. The main elements of handball that affect the result of the match are the following:

- total number of attacks and their effectiveness.

- effectiveness of shots from the front line and back;

- "fast center";

- number of assists;

- number of interceptions.

Data on these criteria improved for all teams in the world elite.

Mr. Dimitrios points out the importance of the" number of interceptions " in the game. We can only imply that the effectiveness of this element in defense is possible only if there is an active contact game in defense.

Murat Bilge notes the analytical significance of shots, the number of attacks, the effectiveness of positional shots, the number of counterattacks, a quick goal, an assist, and the goalkeeper's "saves".

Modern handball became much faster and more dynamic. Based on the results of "top" level tournaments, European teams outperform representatives of other continents in this component of the game. There is not much difference in the statistics for the goalkeepers ' game.

According the research of Willian Ferrari, the success of the Portuguese national team in the international arena is not accidental [8]. Experts concluded that the most significant elements of attacking actions in handball are:

- positional attack;

- counterattack;

- quick center;

- throws from 6-9meters and from 9meters and then;

- collective interaction in 4 zones of the site.

The author approaches the understanding of the most important thing in handball, the goal scored. It is extremely important to determine the situations preceding the opponent's throw in the attack phase in accordance with the score. Track the method of developing an attack from specific game positions:

- rebuilding the team from attack to defense;

- completion of the attack in those positions where the most" vulnerable " place in the opponent's defense;

- attacking actions with active forms of protection, $1+2+3 ; 3+3 ; 2+4 ; 5+1$;

In handball, throw attempts are characterized by the position of the player or the situation in which the throw was made. There are six categories of shots. Three of them are positional (six, extreme, nine) and three are situational, penalty, and counterattack. As the analysis shows, a decrease in the effectiveness of attacks from the front line and an increase in the number of goals from the back line is a consequence of improving the defensive functions of the opposing team. On average, a team makes more than 50 shots per game with an efficiency of $53.2 \%$. However, the number of shots from the six-meter line decreased, but the implementation from the 9-meter line increased. Goals scored from counterattacks account for $18.5 \%$ of the total, and their effectiveness is estimated at $76 \%$. A quick counterattack depends on the ball being blocked by defenders, goalkeepers, rebounds, catching techniques, and passing. The "top-level" commands in this component allow minimum of errors.

A team of authors from Croatia Dragan Milanovic, Marijana Kavala, and Nikolo Faretic pay attention to the consistency and effectiveness of some attacking tactics elements in handball [9]. We studied carefully 90 matches of the Croatian men's championship and identified 19 elements of collective tactics. Attacks, which involve a large number of team players, or group ones are more often used during the game, but they are less effective than individual ones. Attacks directed to the" depth " of the defense are more effective than attacks directed to the width of the attack front. At the same time, the realization of the scoring moment depends on the player who scored the ball, on the partners, the totality of their interactions. Tactics are the essence of the sports game, the management of collective action structures. Use the specific qualities of each player by solving tasks that are compatible with their abilities, in opposition to the other teams. At the same time, our Croatian colleagues are absolutely right to point out that left-handed attacks are more common than right-handed ones, since there are not enough "left-handers" in handball. Coaches take this feature into account when completing teams and building game tactics.

The most effective attack is organized by a group of players and combines the individual skill of an individual handball player. A combination game involving all players is less effective than a simple basic attack.

The quality of defense increases If you analyze the game, the factors leading to victories, evaluating individual and team characteristics, the number of attacks and the effectiveness of shots. According to Taborsky-1996, the effectiveness of attacking actions should be at least $60 \%$. The work of Garcia in 2008 includes the analysis of difference between win and loss among top-level teams. The teams that have a more effective attack, counterattack, implementation of shots from all lines, delivery of the ball to the" depth " of the attack and the number of assists, portend victory. Research shows that winners have an average of five more shots than losers. With an efficiency advantage of $61.31 \%$ versus $48.33 \%$. Now there is a tendency to reduce the effectiveness of attacking actions and increase the effectiveness of defense. However, there is not enough information about which elements of the game in defense are most significant in modern handball. 
Our colleague from the Athens national University, Alexis Karastergios, made a selection for the game of equal teams based on the advantage difference of no more than three goals in previous games [10]. Using the Mann-Whitney $\mathrm{U}$ test, based on a sample of 20 games, he came to the conclusion that the winners outperformed their opponents in the total number of shots in the game, in the number from the 9-meter mark, in the number of vertical passes and the number of counterattacks. The goalkeepers of the winning team blocked $1.8 \%$ more shots. The number of winner 's fouls is $8.6 \%$ higher.The "fast center" has $11-12 \%$ of the team's attacking actions.

M. V. Porokhovskaya came up with a very well-reasoned analysis of competitive activity in handball. She presented a detailed analysis of competitive activities in handball. Research provides valuable information for handball. It is framed in the form of team and individual TTD indices. Effectiveness is determined by the technical and tactical indicators of a highly qualified team in attack:

- number of throws, if effective;

- throws at the counter and their effectiveness;

- the number of shots in a positional attack and their effectiveness;

- number of 7 meter's shots;

- in defense, the number of fouls, violations, 2-minute removals, 7 meter's shots; $\quad$ - the number of blocked shots.

The authors see that there is insufficient development of indicators that characterize competitive activity. The path to victory is achieved by different methods and with different competitors.

To manage the training process and complete the team, you need to:

- availability of a collective game model;

- availability of players matching this model or their ability to reach this level;

- professionalism of the coach, building a long-term plan for the training of the team revealing the potential of players;

- the presence of criteria for competitive activity that determine the effective play of the team.

The score of the team's performance is the points scored and the place in the standings. Attacking actions are indicated by performance and efficiency, the number of goals scored to the total number of shots.

The search for innovations to improve technologies for managing the training process and during competitions in handball is extremely relevant today. The construction of a competitive model in handball, based on scientific and practical research, is a combination of a certain level skill and predicted results. There is no unified methodology for recording analysis and subsequent evaluation of competitive activity parameters.

Table 1. The table of competitive activity indicators of these authors

\begin{tabular}{|c|c|c|c|c|c|c|c|c|c|c|c|c|c|c|}
\hline Criteria & \multicolumn{14}{|c|}{ Surnames } \\
\hline Attacks & 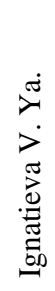 & 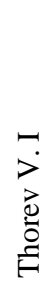 & 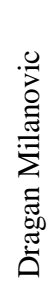 & 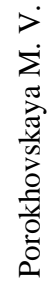 & 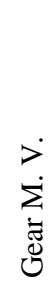 & 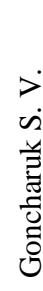 & D & 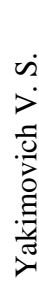 & 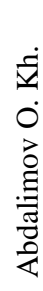 & 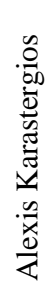 & 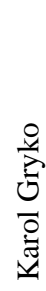 & 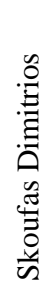 & 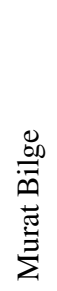 & 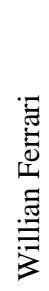 \\
\hline Total attacks & & & + & & & & & & & & & & + & + \\
\hline Positional attack quantity & - & - & + & - & - & - & + & + & + & - & + & - & + & + \\
\hline $\begin{array}{l}\text { The total number of shots per } \\
\text { game /Performance }\end{array}$ & + & + & + & & + & + & + & + & + & + & + & + & + & + \\
\hline $\begin{array}{l}\text { Shots from 6-9 meters / } \\
\text { Performance }\end{array}$ & + & + & + & + & + & & + & + & + & + & + & + & + & + \\
\hline $\begin{array}{l}\text { Shots over } 9 \text { meters / } \\
\text { Performance }\end{array}$ & + & + & + & + & + & & & & + & & + & + & + & + \\
\hline $\begin{array}{l}\text { Shots from extreme positions./ } \\
\text { /performance }\end{array}$ & + & + & + & + & + & + & + & + & + & + & + & + & + & + \\
\hline $\begin{array}{l}\text { Free throws from } 9 \text { meters, incl. } 7 \\
\text { meters / Performance. }\end{array}$ & + & + & + & + & + & + & + & + & + & + & + & + & + & + \\
\hline $\begin{array}{l}\text { Counter-attack throws// } \\
\text { Effectiveness }\end{array}$ & + & + & + & + & - & & + & + & & & + & - & + & + \\
\hline $\begin{array}{l}\text { Quick center / } \\
\text { General/Effectivness }\end{array}$ & - & + & + & + & - & + & & + & - & + & + & & + & + \\
\hline A goal after the group interaction. & + & & - & + & + & - & + & - & - & & - & + & - & + \\
\hline Individually scored goal & + & & + & + & - & + & + & & + & & + & - & - & - \\
\hline Number of 7 meter's & + & & + & & & & & + & + & & - & & + & - \\
\hline Goal pass. & + & & + & + & + & & & + & + & + & + & + & - & - \\
\hline $\begin{array}{l}\text { Transfer under the active } \\
\text { influence of opponents }+/ \text { - }\end{array}$ & - & & - & + & + & & & & - & & - & + & - & - \\
\hline Dribble feint's use $+/-$ & - & & - & + & & & & & + & & - & & - & - \\
\hline
\end{tabular}




\begin{tabular}{|c|c|c|c|c|c|c|c|c|c|c|c|c|}
\hline $\begin{array}{l}\text { Destruction of the opponent for } 2 \\
\text { minutes }\end{array}$ & + & & - & + & & & + & + & - & - & - & - \\
\hline Picking up the ball after an error. & + & & - & & + & & + & - & - & - & - & - \\
\hline The loss that led to the goal. & + & & - & + & - & & & - & + & - & - & - \\
\hline Losing the ball.fishing.broadcasts. & + & & - & + & + & & & + & + & - & - & - \\
\hline \multicolumn{13}{|l|}{ Defence } \\
\hline \multicolumn{13}{|l|}{ Binding, folic } \\
\hline \multicolumn{13}{|l|}{ Exit to throw } \\
\hline Delete for 2 minutes. & + & + & + & + & & & & + & - & & & - \\
\hline $\begin{array}{l}\text { Interaction, a safety in defense }+ \\
/ \text { - }\end{array}$ & + & & + & & & & & - & + & & & + \\
\hline Ball's blocks $+/-$ & + & + & + & + & & & & + & + & & & - \\
\hline bodies' blocks $+/-$ & + & + & - & + & & & & + & + & & & + \\
\hline $\begin{array}{l}\text { Violation with the laterest } 7 \\
\text { meter's. }\end{array}$ & + & + & + & + & & & & - & + & & & - \\
\hline $\begin{array}{l}\text { Number of possession of the ball } \\
\text { interception }\end{array}$ & & + & + & + & & + & & + & + & + & & + \\
\hline Save goalkeeper & & & - & & & & & & - & + & & - \\
\hline Shot total. & & & + & & & & & & + & + & & + \\
\hline
\end{tabular}

Dragan Milanovich and his colleagues analyzed several dozen matches of the OG in London among women's teams and indicated the expected ways of handball development [10]. They manage to do this with difficulty. Analysis of 27 games at the Olympics, 54 confrontations, based on standard indicators: the number of team shots, shots from positions, the effectiveness of interaction between lines, counterattacks, active forms of defense. The winning teams of those matches outperformed their opponents in the efficiency of shots. A special advantage was from the position of extreme and inside forwards. This is an advantage for teams that play aggressively in defense, use active pressing, contact with the attacker to intercept the ball and conduct a quick counterattack. The demands on the players in the back line increased. Along with their superiority in anthropometry, it is necessary to master a wide range of feints, to be able to beat a defender one-on-one, but not to the detriment of interaction with partners. The desire for speed, high mobility, the ability to carry out quick attacks, not allowing the opponent to organize the defense of their own goal, to make a replacement, this is the prospect of developing the game in the coming years.

Our colleague Karol Gryko from the University of physical education in Warshava named after Usef Pilsudsky made forecasts of handball development. How correct and realistic they are today we can already judge [10]. Statistics confirm a decrease in the number of positional attacks by $7.5 \%$, but an increasing number of rapid attacks. At the same time increased the effectiveness of attack by $7.2 \%$. The percentage with a six-meter lines grew by $9.5 \%$, the number of shots from angles increased by $7.3 \%$, and also increased the number of counterattacks by $11.4 \%$. In other words, we see that the speed and dynamics of the game and its density increase. But from this information, we can't say anything about the defensive actions of players in handball. And what counteractions to the growing attacking potential of teams should be taken when defending their own goal.

\section{Conclusions}

The review of the above authors statistics, given in our publication, is based on the statistics and the analysis of technical and tactical actions of the player at the end of the game. It is necessary to increase research using a dynamic approach based on advanced analysis methods. It provides an opportunity to assess the time dynamics of player performance and interactions during a match.

A systematic review can provide useful information about the direction of work for handball analysts.

It is advisable to analyze the game activity in handball in the following areas:

- comparison of winning teams over several seasons (taking into account the stability of the squad or its rotation);

- performance details;

- conducting a large amount of research with a focus on the defensive profile of the team's play;

- analysis of international club handball;

- analysis of statistical reports provided by the organizing committee for the competition;

- use and develop standardized variables for all researchers.

It is necessary to promote the development of systems for gaming activities analyzing not only at the end of the game, but also in the process. At the same time, the interaction of science and practice will be more effectively implemented, expanding cooperation between trainers and scientists.

\section{References}

1. V. A. Ignatieva, V. I. Thorev, Training of handball players at the stage of higher sportsmanship (publishing House: Physical culture, Moscow, 2005)

2. A.S. Kuznetsov, Z.M. Kuznetsova, Russian Journal of Physical Education and Sport, 14(4), 5-7 (2019). DOI: 10.14526/20704798-2019-14-4-5-7 
3. S. A. Sidorchuk, Bulletin of VDU, 2, 71-79 (2016)

4. V. I. Thorev, Scientific notes of the University named after P.F. Lesgaft, 2, 362-365 (2019)

5. V. S. Yakimovich, Scientific notes of the University named after P.F. Lesgaft, 4 (2015)

6. M. V. Parakhovskaya, M. V. Khoptsev, V. A. Galay, Physical Culture and Health, 3, 98-101 (2019)

7. URL: https://www.researchgate.net/publication/330910858 (2019). DOI: 10.7752/jpes. 2019. s1029 citations 0 reads 1695 authors, including:

8. URL: https://www.researchgate.net/publication/330753791 (2018)

9. URL: https://www.researchgate.net/publication/320170459

10. URL: https://www.researchgate.net/publication/328598037 (2017) 